\title{
The epidemiology of subacute sclerosing panencephalitis in England and Wales 1990-2002
}

\author{
C Miller, N Andrews, M Rush, H Munro, L Jin, E Miller
}

Arch Dis Child 2004;89:1145-1148. doi: 10.1136/adc.2003.038489

See end of article for authors' affiliations

.....................

Correspondence to:

Prof. E Miller

Immunisation Department,

Communicable Disease

Surveillance Centre, Health

Protection Agency, 61

Colindale Avenue, London NW9 5EQ, UK; liz.miller@ hpa.org.uk

Accepted

18 February 2004

\begin{abstract}
Aim: To assess the impact of measles/mumps/rubella (MMR) vaccine on the epidemiology of subacute sclerosing panencephalitis (SSPE) in England and Wales.

Methods: Cases of SSPE resident in England and Wales with onset between 1990 and 2002 were reviewed.

Results: A total of 47 cases were identified, 31 male and 16 female. There was an average annual decline of $14 \%$ in SSPE onset over the period, consistent with the decline in notified measles over the last 20 years. A history of measles was present in 35 (median age 1.3 years), the most recent recorded date being 1994; the interval from measles to onset of SSPE ranged from 2.7 to 23.4 years. Four children with a history of receipt of a measles containing vaccine were reported not to have had measles; two of these cases had a brain biopsy, and nucleotide sequence data confirmed wild measles infection. Brain biopsy specimens from a further three cases with a history of measles, of whom two had also had a history of vaccination, showed wild-type strain.

Conclusion: The prevention of endemic circulation of measles virus in England and Wales through the high coverage achieved with MMR vaccine, together with the measles/rubella vaccination campaign of 1994, has resulted in the near elimination of SSPE. However, the recent decline in MMR vaccine coverage, with the associated increase in localised measles outbreaks and cases in young infants, is of concern. It underlines the importance of maintaining high vaccine coverage in order to protect indirectly those most vulnerable to SSPE, namely infants too young to be vaccinated.
\end{abstract}

$\mathrm{S}$ ubacute sclerosing panencephalitis (SSPE) is a rare degenerative disease of the central nervous system causing progressive loss of cerebral function, paralysis, coma, and death. It is the late consequence of measles virus infection, and is associated with viral persistence in brain tissue. ${ }^{1}$

When in 1968 routine measles vaccination with attenuated live virus began in the UK, it was hoped that the expected reduction in measles cases would result in fewer cases of SSPE. It was however thought conceivable, though unlikely, that the attenuated virus might be more prone than the wild form to abnormal persistence, ${ }^{2}$ in which case the incidence of SSPE might actually increase. A Register was therefore set up in 1970 to monitor the incidence of SSPE in England and Wales. ${ }^{3-5}$ A report in $1992^{6}$ showed a significant decline over the period 1970-89, in line with the partial reduction of notified measles over the previous decades; nevertheless SSPE cases continued to occur. In October 1988, measles, mumps, and rubella vaccine (MMR) replaced measles vaccine with a greatly increased acceptance; this was followed by a massive reduction of annual notified measles. The present report covers the years 1990-2002.

\section{SUBJECTS AND METHODS}

The notification methods, criteria for acceptance of cases, and the clinical course have been described previously. ${ }^{6}$ Briefly, cases were ascertained from a variety of sources, including reports from paediatricians through the Surveillance Unit of the Royal College of Paediatrics and Child Health, reports from laboratories to the Communicable Disease Surveillance Centre, and reports from neurologists. The Enteric, Respiratory and Neurological Virus Laboratory (ERNVL) of the Health Protection Agency receives serum and cerebrospinal fluid (CSF) samples from laboratories for diagnostic confirmation. A case is considered confirmed if there is a compatible clinical picture and raised measles antibody titres in serum and CSF, with evidence that the latter is synthesised within the CSF as opposed to serum contamination during CSF collection. All the cases in this report were confirmed by the ERNVL. In March 2002 virology and microbiology laboratories in England and Wales were contacted for reports of SSPE cases diagnosed since 1990; the two reported from this source had already been notified to the Register. Death certificates for relevant categories are received from the Office of National Statistics (ONS); 37 deaths have been reported, all cases being known to the Register.

Information on the age at onset of symptoms, clinical presentation, and progression is sought from the clinician in charge of the case and the general practitioner (GP). The GP is also contacted for a history of measles and/or vaccination (with either measles, MMR, or measles/rubella (MR) vaccine) and date(s) of measles or vaccination. Confirmation of the latter was also sought from the local authority where necessary.

\section{Statistical methods}

The significance of the trend in cases over time was assessed using Poisson regression. The adequacy of the Poisson model was checked by comparing the residual degrees of freedom with the deviance. Differences between proportions were assessed using the $\chi^{2}$ test. The probability of two pregnant cases was calculated from the Poisson distribution. Cox regression was used for the analysis of time from onset to death. The expected number of cases each year from 1990 to 2002 was calculated from data on measles notifications from 1960 to 2001 as well as the previously estimated age specific risk of SSPE following measles infection (risk per $10^{5}=18.0$,

Abbreviations: CSF, cerebrospinal fluid; MR, measles/rubella; MMR, measles/mumps/rubella; SSPE, subacute sclerosing panencephalitis 
$9.8,6.8,4.3,1.7,1.1$ for ages $<1,1,2,3,4,5+$ respectively) and the gamma (index 3 ) distribution for the time from measles to SSPE onset as given in the paper by Farrington. ${ }^{7}$

\section{RESULTS}

From January 1990 to December 2002, 58 cases that met the laboratory criteria for confirmation were notified to the Register. Eleven were foreign nationals who returned home after diagnosis and were therefore excluded. The remaining 47 cases are shown in fig 1 according to year of onset and sex. There is a significant $(p=0.0003)$ downward trend by an average of $14 \%$ per year over the period, from about six cases to one or two per year. In the early 1970s when the SSPE Register was established the annual incidence was about 20 per year. The sex ratio was 1.9:1 with 31 male and 16 female cases. No new cases were reported in 2002.

Two female cases, one with onset in 1999 (aged 19 years) and one in 2000 (aged 15 years) were 30 weeks pregnant. The probability of two cases being diagnosed during pregnancy is only 0.004 based on the age of the female cases and ONS birth rate data. In six cases additional abnormalities were present: encephalocoele, Down's syndrome, epilepsy, congenital toxoplasmosis, family history of tuberose sclerosis, and, in one case, a period of brief learning difficulty in early childhood following a febrile illness. The reporting clinician was asked to give the child's race; of the 47 cases, $10(21 \%)$ were reported as Asian, Indian, Pakistani, or Bangladeshi. This percentage was substantially higher than the proportion of the United Kingdom population under 16 years of age in these ethnic groups in 2001/02 (5.4\%). ${ }^{89}$ Two cases occurred in one of a pair of twins, the other twin in each pair being unaffected.

\section{SSPE onset and death}

The precise age at onset was sometimes difficult to define and largely reflected the age at which the patient first sought medical help. The age at onset of SSPE ranged from 4 to 26 years (median 12.5). The mean age at onset increased over the study period by an average of 4.4 months a year (standard error 2.4 months). Although this difference did not reach statistical significance $(p=0.07), 67 \%$ of cases with onset from 1994 to 2002 were over the age of 12.5 years compared with $38.5 \%$ in the first four years. There was no difference in age of onset by sex or ethnicity.

By March 2003, 37 of the 47 cases had died; of the 10 still alive, six had survived since onset for over six years. The course from onset to death varied from 1 month to over 12 years with a median of 2.7 years. Of the two girls who were 30 weeks pregnant at the time of onset, one died after five months and the other is still alive after three years. Both babies were apparently normal at birth and during their early lives, although one had had a convulsion at 6 months.

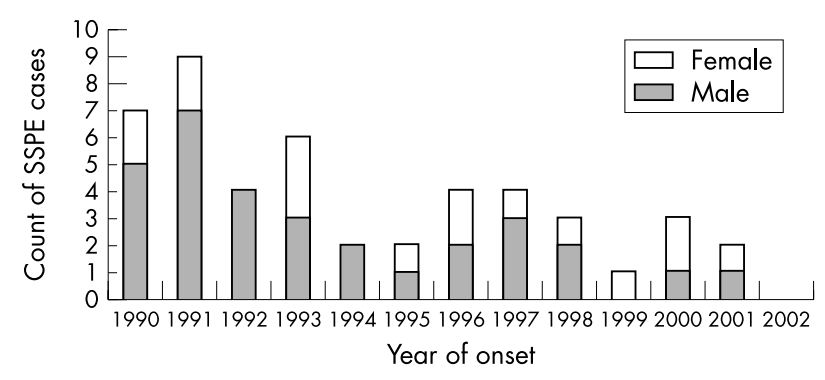

Figure 1 SSPE cases by year of onset and gender.

\section{History and age of measles}

Measles was recorded for 35 of the 47 cases, 33 with the date; eight were reported not to have had measles, and for four there was no information. The most recent recorded dates of measles were three in 1988 and one each in 1993 and 1994. The median age of measles was 1.32 years (range 0.4-6 years). In $64 \%(21 / 33)$ measles occurred under the age of 2 years, and in $24 \%$ (8/33) under 1 year. Measles preceding SSPE occurred at a significantly younger age than in the general population $(p=0.001)$, in whom only $21 \%$ had measles under 2 and $10 \%$ under 1 (fig 2). The median interval from measles to the onset of SSPE was 9.7 years; the range was from 2.7 to 23.4 years. This interval did not vary significantly by sex, year of onset, age at measles infection, or ethnicity.

As was seen previously, ${ }^{6}$ in those infected with measles at a younger age the course of SSPE from onset to death was longer $(p=0.009)$. This is shown by a median of 4.2 years in those infected under the median age at infection of 1.32 years compared to 1.9 years in those infected above the median age.

\section{Vaccination with measles containing vaccines}

Vaccination was reported in 23 cases (49\%); 17 had had a single dose of either measles, MMR, or MR vaccine, five had had two doses of a measles containing vaccine, and one had had three doses. For six patients the vaccination status was unknown; 18 were reported not to have been vaccinated, of whom 14 had recorded measles.

Of the 23 cases with a vaccination history, 18 also had a history of measles; in 12 measles occurred before vaccination, in two after vaccination, in two around the same time, and in two the timing was unclear due to a missing vaccination date. In four vaccinated cases there was no history of measles and in one the measles history was unknown.

\section{Brain biopsy}

Brain biopsy was performed on a total of five patients, all of whom had wild strain measles virus identified by nucleotide sequencing of the $\mathrm{H}$ and $\mathrm{N}$ genes. ${ }^{10}$ These included two of the four with a history of vaccination but no history of measles (table 1). All five cases were born between 1979 and 1985 and had biopsy specimens taken between 1997 and 2000; all measles nucleotide sequences were consistent with the wild strains known to be circulating in the 1980s and 1990s. ${ }^{10}$

\section{Comparison of the observed and expected number of cases from 1990 to 2002 based on the analysis of the 1970-89 SSPE data ${ }^{7}$}

The observed and expected numbers are given in fig 3. This shows a good fit and a total number of 39 cases expected, slightly fewer than the observed total of 47 . To examine the

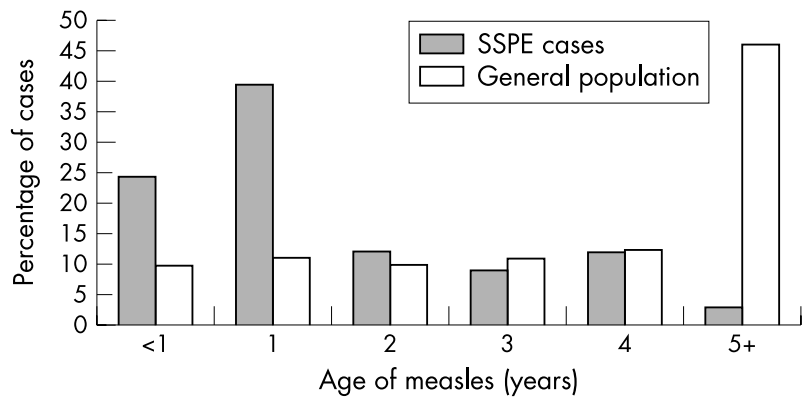

Figure 2 Age distribution of measles onset in SSPE cases and the general population using notification data from 1970 to 1994 in England and Wales. 
Table 1 Details of the five SSPE cases who had a brain biopsy, all of whom had wildtype virus identified

\begin{tabular}{llll}
\hline Case/date of birth & Measles disease history & Vaccination history & SSPE onset \\
\hline 1. 1984 & None & MR vaccine January 1995 & 1999 \\
2. 1985 & None & Measles vac 1988, MMR 1989 & 1994 \\
3. 1979 & 1980 & Not vaccinated & 2000 \\
4. 1981 & 1983 & MR 1994 & 1997 \\
5. 1982 & 1983 & MR 1994 & 1998 \\
\hline
\end{tabular}

possibility of MMR giving rise to SSPE, the observed number of cases from 1992 to 2002 can be compared to the expected number, which is based on the risk being only from measles infection. The observed number of 31 is very close to the expected number of 29. Note that the period from 1992 to 2002 was chosen because any MMR effect would not become apparent until 1992, given its introduction in October 1988 and the delay to SSPE onset.

\section{DISCUSSION}

This study shows that the reduced incidence of measles brought about by vaccination has caused the almost total disappearance of SSPE in England and Wales. From 1968, when measles vaccine was introduced, annual measles notifications fell to about a sixth of the pre-vaccine figures but still exceeded 100000 in most years. This was due to the poor uptake of measles vaccine, which never rose above $70 \%$. When MMR vaccine replaced it in October 1988, acceptance was quickly reached and, until recently, remained above $90 \%$. Following the national MR vaccination catch-up programme for 5-16 year old children in 1994, enhanced laboratory based surveillance of measles was established and has provided strong evidence that endemic circulation of measles in the UK has been interrupted. ${ }^{11}$ However, because of the delayed onset of SSPE, a few cases in older children and young adults may occur in the near future as a result of infection acquired in the 1980s and early 1990s. The increase in the age at onset observed over the study period is consistent with the recent cases arising from infection acquired in the 1980s and early 1990s.

While the recent drop in MMR coverage in the UK has not yet allowed the endemic circulation of measles to be reestablished, there have been a number of localised outbreaks in 2002 in groups declining MMR vaccine because of safety fears. ${ }^{12}$ A disturbing feature of these localised outbreaks has been the involvement of infants under 12 months of age. Of the 308 laboratory confirmed measles cases in England and Wales in 2002, 33 (11\%) were infants under a year, ${ }^{13}$ this compares with a total of 40 confirmed cases in infants in the

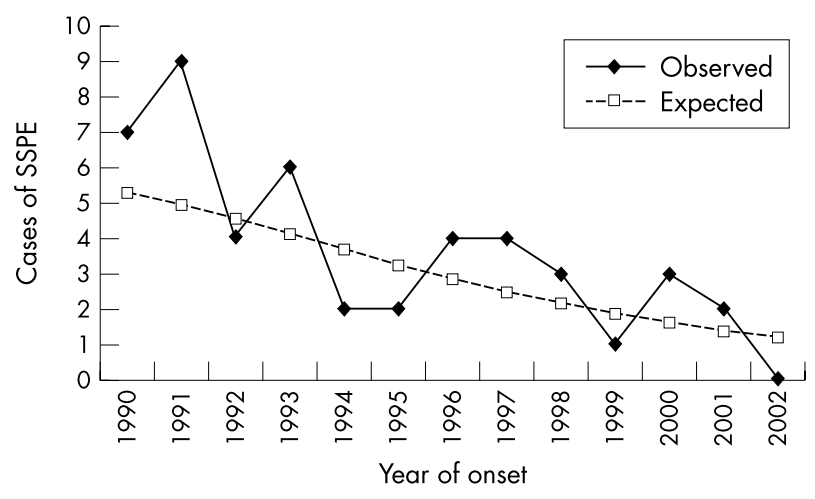

Figure 3 Observed and expected numbers of SSPE cases by year of onset. seven years from 1995 to 2001. As in our previous SSPE report, ${ }^{6}$ measles infection in SSPE cases occurred at a significantly younger age than in the general population. In the current study the last two measles cases preceding SSPE occurred in 1993 and 1994, both in children under 1 year. Current advice is to offer MMR vaccine to infants from 6 months of age in the event of a localised outbreak, with revaccination at 12-15 months of age and again at school age. Infants aged 6-8 months in whom maternal antibody may have waned should be offered post-exposure prophylaxis with normal immunoglobulin if they are household contacts of a confirmed case. ${ }^{14}$

The good fit of the observed number of cases from 1990 to 2002 to the number expected based on notifications of measles, the previously derived age specific risk estimates, and gamma delay distribution ${ }^{7}$ provide validation of these estimates; these gave an overall risk of SSPE following measles of 1 per 25000 and a delay distribution with a mean of 7.4 years. The risk of SSPE following measles in the first year of life is, however, substantially greater at around 1 in $5500 .{ }^{6}$ Based on an annual birth cohort of 650000 and the overall risk of SSPE of 1 per 25000 measles cases, the expected number of SSPE cases from 1990 to 2002 in the absence of vaccination would have been over 300, compared with the observed number of 47 .

The higher than expected proportion of cases who were Asian is consistent with the earlier study which found that $22 \%$ of the cases with onset between 1985 and 1989 were Asian. ${ }^{6}$ The reason for this increased risk of SSPE in this group is unclear but may reflect genetic differences, environmental factors, or differences in measles exposure.

The surveillance shows that the decline in the incidence of SSPE following the introduction of measles vaccine has continued following the change to MMR vaccine in October 1988. Since most cases have an interval of over three years from measles virus exposure to onset of SSPE, any possible MMR vaccine attributable cases would be expected to occur in the period from 1992 onwards. However, the number of cases with onset from 1992 onwards closely fits the number predicted based on the assumption that no cases are vaccine attributable. The virus present in the brain in two SSPE cases without a history of measles disease but who had been vaccinated (one with MR at 11 years and one with measles vaccine at 3 years and MMR vaccine at 4 years) was confirmed as wild-type. In such cases, as in the four cases with neither a history of wild measles nor vaccination, measles infection must have occurred but not been recognised, possibly because it occurred in the first year of life when the disease may be mild and easily missed. The diagnosis of SSPE should therefore still be considered in the absence of a history of measles.

A reduction in SSPE following the introduction of vaccination has been reported from the United States, ${ }^{15}$ Poland, ${ }^{16}$ the Netherlands, ${ }^{17}$ and in the Middle East. ${ }^{18}{ }^{19}$ Our study confirms the impact of vaccination and shows that the elimination of SSPE is possible through the use of MMR vaccine. It also underlines the importance of maintaining 
high levels of vaccine coverage in order to protect indirectly those most vulnerable to SSPE, namely infants too young to benefit from the direct protective effects of vaccination. Surveillance is continuing in England and Wales in order to monitor the effect of the recent decline in MMR vaccine coverage on progress towards elimination of SSPE.

\section{ACKNOWLEDGEMENTS}

We thank Dr Shunmay Yeung for her help with the follow up and virological investigation of the cases. We also thank the general practitioners, paediatricians, virologists, and neurologists who provided information.

\section{Authors' affiliations}

C Miller, M Rush, H Munro, E Miller, Immunisation Department, Communicable Disease Surveillance Centre, Health Protection Agency, 61 Colindale Avenue, London NW9 5EQ, UK

N Andrews, Statistics, Modelling and Economic Evaluation Department Communicable Disease Surveillance Centre, Health Protection Agency, 61 Colindale Avenue, London NW9 5EQ, UK

L Jin, Enteric, Respiratory and Neurological Virus Laboratory, Division of Specialist and Reference Microbiology, Health Protection Agency, 61 Colindale Avenue, London NW9 5EQ, UK

\section{REFERENCES}

1 Horta-Barbosa L, Fuccillo DA, Sever JL, et al. Subacute sclerosing panencephalitis: isolation of measles virus from a brain biopsy. Nature 1969;221:974

2 Dick G. Register of cases of subacute sclerosing panencephalitis. BMJ 1973;3:359.

3 Dick G. Register of cases of subacute sclerosing panencephalitis (SSPE). BMJ 1975;3:238.
4 Bellman M, Dick G. Register of cases of subacute sclerosing panencephalitis. BMJ 1977; 1:430.

5 Bellman M, Dick G. Surveillance of subacute sclerosing panencephalitis. BMJ 1980;281:393.

6 Miller C, Farrington CP, Harbert K. The epidemiology of subacute sclerosing panencephalitis in England and Wales 1970-1989. Int J Epidemiol 21, 5:998.

7 Farrington CP. Subacute sclerosing panencephalitis in England and Wales: transient effects and risk estimates. Stat Med 1991;10:1733-44.

8 http://www.statistics.gov.uk/cci/nugget.asp?id = 273 .

9 http://www.statistics.gov.uk/cci/nugget.asp?id $=272$.

10 Jin L, Beard S, Hunjan R, et al. Characterization of measles virus strains causing SSPE: a study of 11 cases. J Neurovirol 2002;8:335-44.

11 Vyse AJ, Gay NJ, White JM, et al. Evolution of surveillance of measles, mumps and rubella in England and Wales-providing the platform for evidence based vaccination policy. Epidemiol Rev 2003;24:125-36.

12 Ramsay ME, Jin L, White J, et al. The elimination of indigenous measles transmission in England and Wales. J Infect Dis 2003; 187:S198-207.

13 Public Health Laboratory Service. Laboratory confirmed cases of measles by region and age-England and Wales, 1996-2002. http://www.hpa.org.uk/ infections/topics_az/measles/data_reg_age.htm

14 PHLS. The immunoglobulin handbook. April 2002, p. 5 Measles. http:// www.hpa.org.uk/infections/topics_az/immunoglobulin/ immunoglobulinHandbook.

15 Dyken PR, Cunningham SC, Ward LC. Changing character of subacute sclerosing panencephalitis in the United States. Pediatr Neurol 1989;5:339-41.

16 Naruszewicz-Lesiuk D, Wieczorkiewicz M, Iwinska-Buksowicz B, et al. Prospects of measles and subacute sclerosing panencephalitis (SSPE) elimination in Poland. Neurol Neurochir Pol 1999;33:653-63.

17 Beersma MF, Galama JM, Van Druten HA, et al. Subacute sclerosing panencephalitis in the Netherlands-1976-1990. Int J Epidemiol 1992;21:583-8.

18 Zilber N, Rannon L, Alter M, et al. Measles, measles vaccination, and risk of subacute sclerosing panencephalitis (SSPE). Neurology 1983;33:1558-64.

19 Modarres SH, Ghofrani M. The epidemiology of subacute sclerosing panencephalitis (SSPE) in Northern Iran. Irn J Med Sci 1998;23:29.

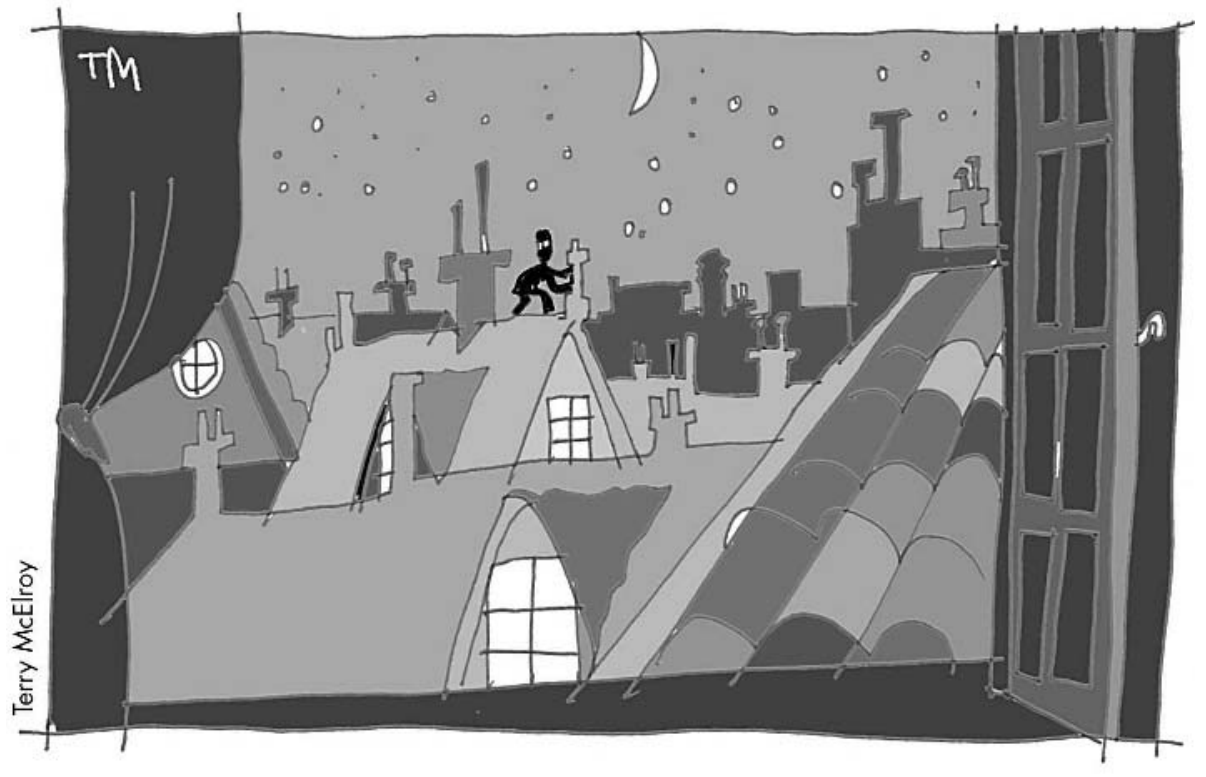

